\title{
Reconnection of a kinking flux rope triggering the ejection of a microwave and hard X-ray source
}

\author{
II. Numerical Modeling
}

\author{
B. Kliem ${ }^{1,2,3}$, M. G. Linton ${ }^{3}$, T. Török ${ }^{4}$, M. \\ Karlický ${ }^{5}$
}

Received 24 March 2010; accepted 7 July 2010

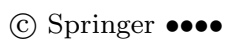

\begin{abstract}
Numerical simulations of the helical $(m=1)$ kink instability of an arched, line-tied flux rope demonstrate that the helical deformation enforces reconnection between the legs of the rope if modes with two helical turns are dominant as a result of high initial twist in the range $\Phi \gtrsim 6 \pi$. Such reconnection is complex, involving also the ambient field. In addition to breaking up the original rope, it can form a new, low-lying, less twisted flux rope. The new flux rope is pushed downward by the reconnection outflow, which typically forces it to break as well by reconnecting with the ambient field. The top part of the original rope, largely rooted in the sources of the ambient flux after the breakup, can fully erupt or be halted at low heights, producing a "failed eruption." The helical current sheet associated with the instability is squeezed between the approaching legs, temporarily forming a double current sheet. The leg-leg reconnection proceeds at a high rate, producing sufficiently strong electric fields that it would be able to accelerate particles. It may also form plasmoids, or plasmoid-like structures, which trap energetic particles and propagate out of the reconnection region up to the top of the erupting flux rope along the helical current sheet. The kinking of a highly twisted flux rope involving leg-leg reconnection can explain key features of an eruptive but partially occulted solar flare on 18 April 2001, which ejected a relatively compact hard X-ray and microwave source and was associated with a fast coronal mass ejection.
\end{abstract}

Keywords: Magnetohydrodynamics (MHD) - instabilities - Sun: coronal mass ejections (CMEs) - Sun: flares - Sun: radio radiation

\footnotetext{
1 Universität Potsdam, Institut für Physik und Astronomie, 14476 Potsdam, Germany (e-mail: bkliem@uni-potsdam.de)

2 University College London, Mullard Space Science

Laboratory, Holmbury St. Mary, Dorking, Surrey, RH5 6NT $\mathrm{UK}$

3 Naval Research Laboratory, Space Science Division,

Washington, DC 20375, USA

4 LESIA, Observatoire de Paris, CNRS, UPMC, Université

Paris Diderot, 5 place Jules Janssen, 92190 Meudon, France

5 Astronomical Institute, Academy of Sciences of the Czech

Republic, 25165 Ondřejov, Czech Republic
} 


\section{Introduction}

When a line-tied, arched magnetic flux rope becomes unstable with respect to the helical kink mode, i.e., to displacements with azimuthal wavenumber $m=1$, several effects occur which are relevant to the understanding of solar eruptions (Sakurai, 1976, Fan and Gibson, 2003 Török, Kliem, and Titov, 2004). First, the apex of the rope will ascend if the initial perturbation points upward, which is usually the case for solar eruptions due to a slow rise of the structure prior to the main acceleration. Second, the ensuing fast rise is initially exponential or a power law close to an exponential, as observed to be typical for fast coronal mass ejections (CMEs) (Vršnak, 2001; Schrijver et al., 2008a). Third, the rope will writhe by the conversion of twist helicity into writhe helicity. This leads to a rotation of the apex about the direction of ascent and forms a characteristic inverse gamma shape or O shape (depending on the perspective of the observer). Such shapes are often observed and have been taken as evidence for the occurrence of the instability (e.g., Rust, 2003; Ji et al., 2003, Romano, Contarino, and Zuccarello, 2003, Rust and LaBonte, 2005, Török and Kliem, 2005, Fan, 2005, Zhou et al., 2006 Gilbert, Alexander, and Liu, 2007, Cho et al., 2009). Fourth, two current sheets will be formed and exponentially steepened, so that the onset of magnetic reconnection is likely: a vertical (flare) current sheet under the rope resulting from the rope's rise; and a helical current sheet, wrapped around the rope and passing over the rope's apex, created as a consequence of the helical displacement. Reconnection in these current sheets influences the eruption strongly. It leads to a variety of different dynamical behaviors, including ejective and confined eruptions (Török and Kliem, 2005) as well as the break-up and reformation of the rope, which we will address in the present paper. Fifth, sigmoids are likely to form, since the field lines that thread either of the current sheets are sigmoidal in projection (Titov and Démoulin, 1999; Fan and Gibson, 2003; Kliem, Titov, and Török, 2004 Gibson et al., 2004). The orientation of the sigmoidal field lines underneath the flux rope, where sigmoids are known to form, correlates with the chirality of the field in agreement with the observations (Rust and Kumar, 1996; Pevtsov, Canfield and McClymont, 1997: Green et al., 2007).

The literature reports a number of events that suggest the occurrence of the helical kink instability through observational evidence for both the existence of considerable twist (exceeding one field line turn about the axis of the structure) and the development of writhe in the course of the eruption. Examples are the events of 19 July 2000 (Romano, Contarino, and Zuccarello, 2003), 18 August 1980 (Vršnak et al., 1993), and 12 June 2003 (Liu and Alexander, 2009); see also the systematic study by Vršnak, Ruždjak, and Rompolt (1991).

In spite of such considerable principal agreement and support, it is possible that the instability actually occurs less frequently than indicated by the above observations, because there exists a second physical process that can cause the writhing of a rising flux rope into helical shape. As has been pointed out by Isenberg and Forbes (2007), the shear field component of the ambient field (pointing along the photospheric polarity inversion line), crossed with the vertical current component in the legs of a flux rope which is displaced upwards out of equilibrium, gives a sideways pointing Lorentz force on the flux rope 
legs. This force yields a writhing in the same direction as the kink-mode-driven writhing for any process that lifts the rope out of equilibrium. Basically, the same observational consequences as listed above can be expected, including the reduction of any possibly existing initial twist into the developing writhe, due to helicity conservation.

Therefore, to evaluate the relevance of the helical kink instability for solar eruptions, it is important to distinguish between the two possible causes of the writhing into helical shape. The direct approach - computing the coronal field by extrapolation of vector magnetograms and comparing the obtained twist to the threshold of the helical kink mode - is still hardly practicable because present vector magnetograms and nonlinear force-free extrapolation codes do not appear to have reached the degree of consistency required for a reliable computation (Metcalf et al., 2008, Schrijver et al., 2008b), and because a comprehensive parametric study of the instability threshold has not yet been completed (although some knowledge is already available, see Török, Kliem, and Titov, 2004 and the references therein). Hence, it is of interest to identify features that permit an observational discrimination.

The writhing by the shear field component has the property that the sideways pointing Lorentz force acting on the flux rope legs has the same sign all along one leg, and the opposite sign along the other leg; it changes sign only at the apex. This implies that the displaced flux rope cannot develop more than one helical turn. In terms of a normalized axial wavenumber, $k^{\prime}=k l / 2 \pi=l / \lambda$, where $l$ is the length of the flux rope and $\lambda$ is the wavelength of the perturbation, this can be expressed as $k^{\prime} \leq 1$.

In contrast, the helical kink instability can have any wavenumber in principle. Its dominant wavenumber is set by the twist $\Phi=l B_{\phi} / r B_{z}=2 \pi N$, where $N$ is the number of field line turns in the flux rope, in general averaged over its cross section. Stability analyses indicate that maximum growth occurs in the range $\lambda \sim(1-2) l_{\mathrm{p}}$, where $l_{\mathrm{p}}=l / N$ is the pitch length, the axial length required for one field line turn (Linton, Longcope, and Fisher, 1996: Linton et al., 1998). For a force-free flux rope with uniform radial twist profile, for example, maximum growth occurs for $\lambda=2 l_{\mathrm{p}}$. In this case, the $k^{\prime} \sim 1$ and $k^{\prime} \sim 2$ modes are dominant for $\Phi \sim 4 \pi$ and $\Phi \sim 8 \pi$, respectively, so that the transition between them occurs in this range of twist values. Such high values render the occurrence of $k^{\prime}>1$ modes significantly less likely than the occurrence of single helical modes. For twist profiles that lead to maximum growth closer to $\lambda=l_{\mathrm{p}}$, the transition will occur between twists of $2 \pi$ and $4 \pi$, i.e., closer to the kink instability threshold, so it could be more common. However, it is not clear whether such nonuniform profiles are often formed in the solar atmosphere. The observations of erupting filaments rather tend to indicate $k^{\prime} \lesssim 1$ helical modes in the majority of events.

If modes $k^{\prime}>1$ are observed, the helical kink instability is implied. Projected onto the plane of the sky, this manifests itself as the occurrence of more than one inflection point of the kinked flux tube. For events near Sun center, an S shape with an overturn at one or both ends results (see the kinking flux rope in Figure 2 below at $t=11$ for an illustration). In fortunate cases the effect is apparent even in limb events, for example in the failed filament eruption of 27 May 2002, which showed an inflection point in the middle of the front 
leg (see the middle panels of Fig. 1 in Török and Kliem, 2005). If $k^{\prime}$ is clearly larger than unity, then the multiple helical shape is relatively clearly indicated for any perspective; an example is the 19 July 2000 filament eruption analyzed in Romano, Contarino, and Zuccarello (2003). Such direct observations of the helical mode with $k^{\prime}>1$ are not very numerous, however.

Another possible consequence of $k^{\prime}>1$ mode dominance is an approach of the rising flux rope's legs so that they can come into contact and begin to reconnect. Such an evolution is not triggered by a $k^{\prime} \leq 1$ helical displacement, which rather causes the legs of the flux rope to move apart. It is expected to have distinct observational consequences. The reconnection can cause the breakup of the original flux rope as suggested by Cho et al. (2009). Furthermore, the simulations in Section 2 demonstrate that such break-up can lead to the reformation of a (less twisted) flux rope from the two bottom parts of the original rope, which facilitates the quick reformation of a filament after an eruption. Finally, the energetic particles produced by the reconnection can have unique radiation signatures if the helical current sheet is involved. This was found in the companion paper to the present study (Karlický and Kliem, 2010, hereafter Paper I), which analyzes the observations of an eruptive flare on 18 April 2001 in NOAA Active Region (AR) 9415. This partly occulted eruption formed a rapidly ascending microwave source of strongly helical (inverse gamma) shape (shown also in Figure 7 below) and presents evidence for the acceleration of nonthermal particles at or near the crossing point of the flux loop legs (as seen in the projection onto the plane of the sky). Moreover, the particles were efficiently trapped in superimposed moving compact sources, which are suggestive of plasmoids formed by reconnection between the legs and moving in the helical current sheet.

In the present paper, we demonstrate the reconnection between the legs of a kinking flux rope using magnetohydrodynamic (MHD) simulations. We estimate the minimum twist enabling this process and study its dynamics. The simulation results are then discussed in relation to the observations of the eruptive flare on 18 April 2001 which were found in Paper I to be suggestive of such reconnection.

\section{Helical Kink Instability of a Highly Twisted Flux Rope}

The zero-beta, ideal MHD simulations presented in this paper are all largely similar to those in Török and Kliem (2005). They use the force-free equilibrium of a toroidal flux rope by Titov and Démoulin (1999) as initial condition and employ similar numerical parameters (a Cartesian box of size $10 \times 10 \times 20$, discretized by a stretched grid of resolution $\Delta=0.03$ in the central part, and very similar small viscosity and weak spatial smoothing, as required to maintain numerical stability). Magnetic reconnection occurs in these simulations as a consequence of numerical diffusion where current layer(s) steepen in response to the development of ideal MHD instability.

Most geometrical parameters of the rope, as well as the normalization, are also set as in these previous simulations: major torus radius $R=1.83$, depth of torus center $d=0.83$, distance of the sources of the external poloidal field (the 


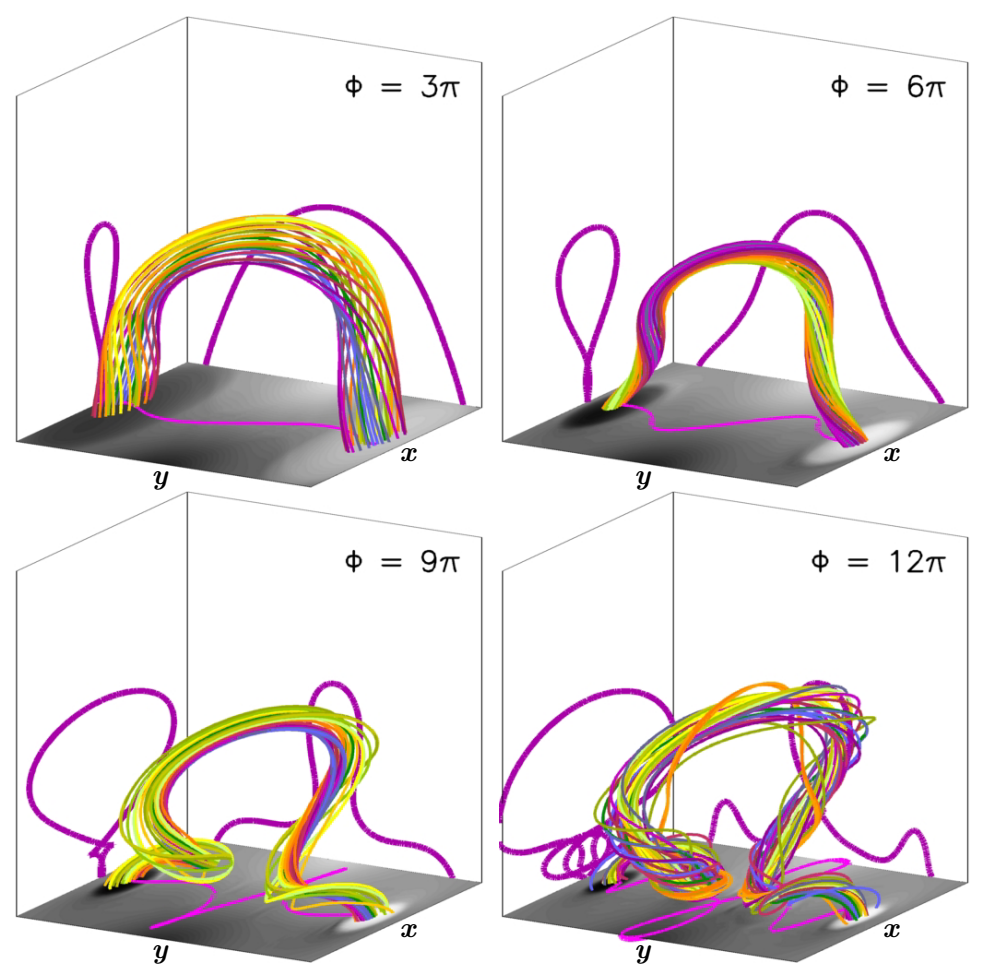

Figure 1. Flux rope shape for a range of initial twist values and uniform apex height of twice the initial apex height (reached at $t=34,19,13$, and $12 \tau_{A}$ in the simulations). The volume $1 \times 4 \times 4$ is shown; the stretching in the $x$ direction helps to visualize the helical shapes. All field lines are started from circles of radius $0.2 a$ centered at the footpoints of the flux rope. The orthogonal projections show the magnetic axis of the rope.

field components perpendicular to the flux rope axis) $L=0.83$. The line current in the model, which produces the external toroidal field, is reduced by a factor 20 in comparison to the simulation of a failed eruption in that previous paper, to enable eruptive behavior (Roussev et al., 2003; Török and Kliem, 2005). The toroidal field component points along the flux rope axis and is essentially also the shear field component, since the polarity inversion line of the field in the bottom plane runs approximately parallel to the flux rope under its apex. The external field is thus mainly poloidal, with the ratio between toroidal and poloidal components at the flux rope apex being $B_{\text {et }} / B_{\text {ep }}=0.075$. Consequently, any development of writhe is nearly exclusively due to the helical kink mode. Gravity is neglected, and the initial density is derived from the initial magnetic field by $\rho_{0}=\left|\mathbf{B}_{0}(\mathbf{x})\right|^{3 / 2}$, to model a corona with an Alfvén velocity that decreases slowly with distance from the flux concentrations. Initially, the flux rope lies in the plane $\{x=0\}$, and its field strength is normalized to unity at the apex, $B_{0}=|\mathbf{B}(0,0,1,0)|=1$. This gives an initial Alfvén velocity $V_{A 0}=B_{0} \rho_{0}^{-1 / 2}=1$ at the apex, which serves as the velocity unit and defines the time unit through $\tau_{A}=1 / V_{A 0}$. 
The average twist of the flux rope is varied between the simulations in the range $\Phi=(3-16) \pi$ (in steps of $1 \pi$ between 3 and $10 \pi$ ) by varying the minor torus radius between $a=0.60$ and $a=0.11$. All these equilibria are unstable with respect to the helical kink mode. See Török, Kliem, and Titov (2004) for the calculation of the average twist, for the overall evolution of the instability in such equilibria, in particular for the formation of the flare current sheet and the helical current sheet, and for a parametric study of the growth rate.

For equilibria of progressively higher twist, the axis of the kinked flux rope shows progressively higher values of the number of helical turns, with the transition to a dominance of the double helical mode $\left(k^{\prime} \sim 2\right)$ occurring for $\Phi \approx 6 \pi$. As a consequence, the helical deformation of the rope legs leads to a progressively smaller separation between the legs under the rising rope apex (Figure 1). This facilitates the onset of reconnection between the legs. For much higher twists $(\Phi \gtrsim 12 \pi)$, higher axial modes $\left(k^{\prime} \gtrsim 3\right)$ dominate initially, but the upward expansion of the flux rope leads to the dominance of the double helical perturbation in the nonlinear evolution of the instability, an effect we will address in a future study.

The twist value at the transition between the regimes of single and double helical mode dominance depends not only upon the radial twist profile of the rope as discussed in the Introduction, but also on the height profile of the overlying potential field. This dependence will be studied in more detail in a future paper. Our finding of $\Phi \gtrsim 6 \pi$ for double helical mode dominance may be compared with the simulation of the helical kink instability in a helmet streamer-like configuration above a highly twisted flux rope in Birn, Forbes, and Hesse (2006). The erupting top part of their flux rope had twists in the range $\approx(9-12) \pi$ and it clearly showed the double helical shape of a $k^{\prime} \approx 2$ perturbation with an axis shape similar to the $\Phi=9 \pi$ snapshot in Figure 1. Unfortunately, no statement was given whether the legs did begin to reconnect in the later phases of the evolution.

\subsection{Reconnection in the $m=1, k^{\prime} \sim 2$ Helical Kink}

In the following we discuss one run of the series in more detail, taken to be the run with $\Phi=9 \pi$. This run shows a clear dominance of the double helical mode, which we intend to relate to the observations of the 18 April 2001 eruption. While this event motivated our study, we do not attempt to model the observed source shape, apex trajectory, and height of the crossing point very closely in this section. The purpose of the simulation is primarily to demonstrate that the legs of the rope reconnect if the twist is sufficiently high, with the apex continuing to rise at high speed and the region of leg-leg reconnection not participating in the fast rise. Quantitative comparisons between simulation and observation are given in Section 2.2 and in Paper I.

Figure 2 presents snapshots of field lines in the core of the flux rope which show the initial condition, the evolution of the double helical $k^{\prime} \sim 2$ kink perturbation, and the approach and interaction of the rope legs. The reconnection of the outer flux layers of the rope begins between the second and third snapshot pair and the corresponding perturbation has propagated into the core of the rope by the 

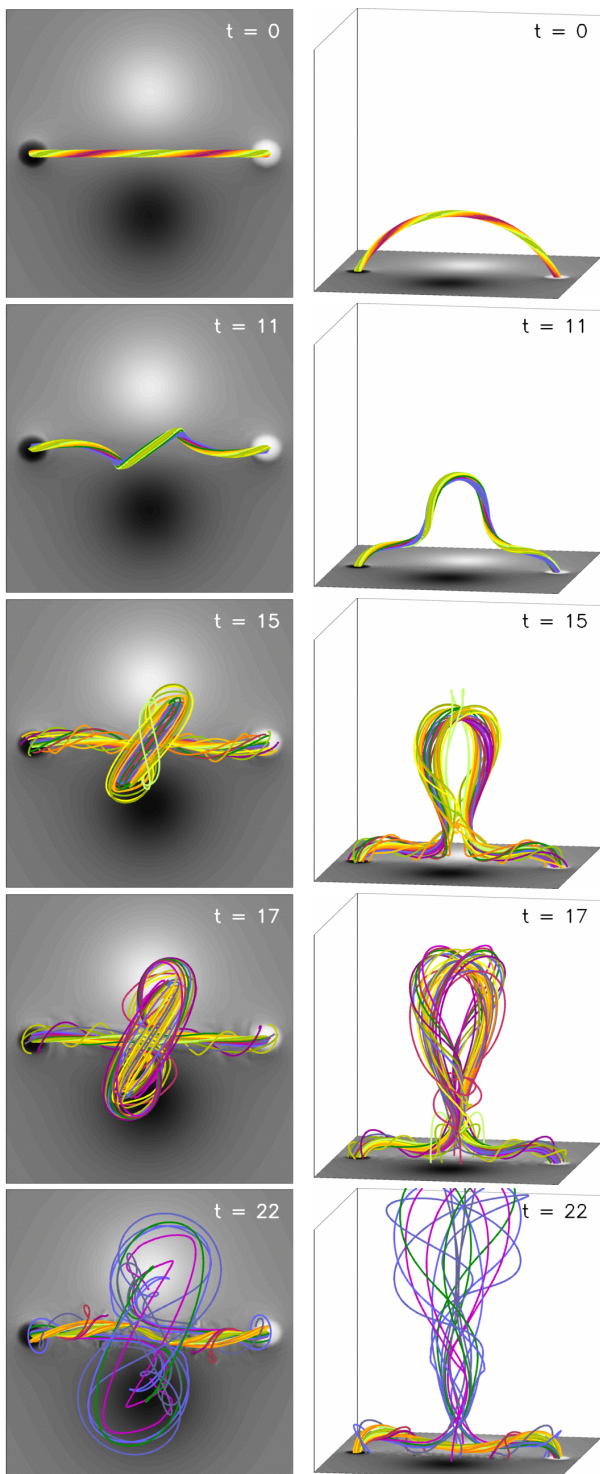

Figure 2. Field lines in the core of a kink-unstable flux rope with initial average twist $\Phi=9 \pi$ (started at circles of radius $r=0.2 a$ centered at each footpoint of the magnetic axis of the rope) in top and perspective views. This shows the evolution of the double helical $\left(k^{\prime} \sim 2\right)$ perturbation, which leads to the approach and subsequent reconnection of the flux rope legs. A new low-lying, less twisted flux rope is formed (bottom panels). The inner part (of size $4 \times 4 \times 4)$ of the simulation box is displayed, including the magnetogram, $B_{z}(x, y, 0, t)$.

time of the third snapshot pair, distorting the field lines, although the core still has the original field line connections. The final snapshot pair shows the system when most of the flux in the original rope has reconnected, resulting in the 

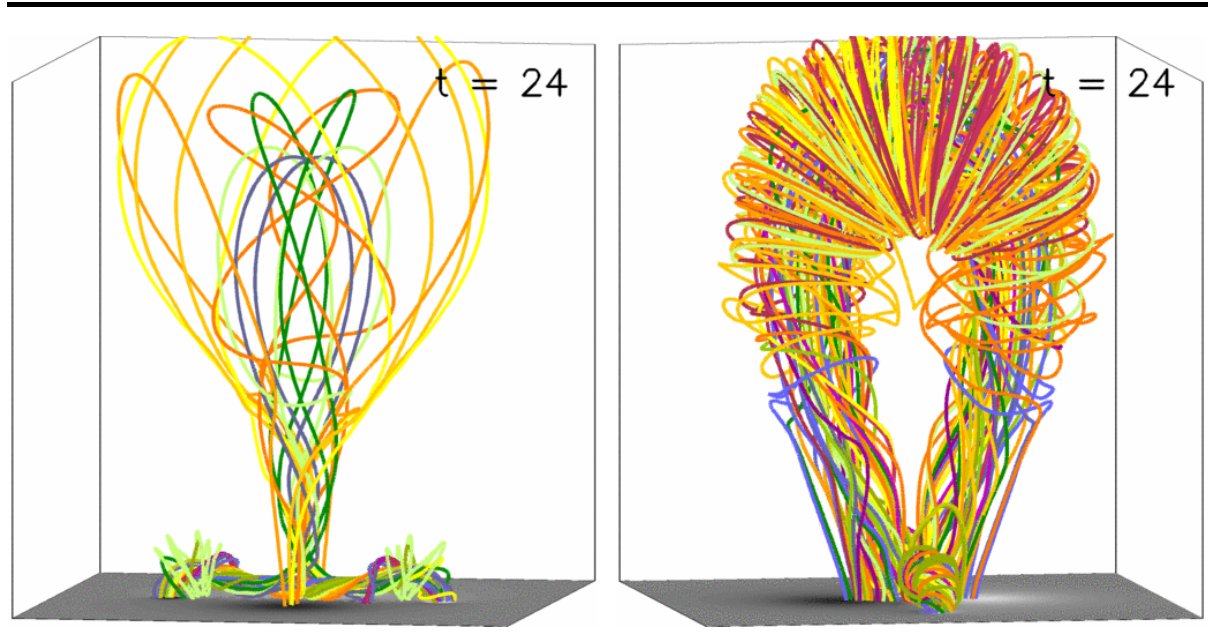

Figure 3. Left: Field lines started at the same positions as in Figure 2 at a later time, after the original flux rope legs have reconnected. A new flux rope is formed below the reconnection region, which remains at low heights $(z \lesssim 1)$, while the top part of the original rope continues the upward expansion. Right: Field lines started in the top part of the rising flux at the same time, showing the flux rope structure and the new magnetic connections to the sources of the originally overlying flux. This is supplemented by some field lines started at the flux rope footpoints. The inner subvolume of size $6^{3}$ is shown. The views differ by a rotation of $70^{\circ}$ about the vertical $(z)$ axis.

break-up of the original rope and reformation of an only weakly kinked, still twisted flux rope at low heights.

Both the rise of the rope's upper part and the reconnection at low heights continue subsequently. Figure 3 illustrates that the rising flux still has the character of a flux rope, although its average twist is reduced and although it becomes increasingly rooted in the ambient flux, which is obviously involved in the reconnection that breaks up the original rope. The downward reconnection outflow pushes the newly formed flux rope toward the bottom boundary, enforcing further reconnection with the ambient flux. Eventually, the newly formed rope breaks up as well into two flux bundles that connect each footpoint of the original rope to the corresponding external flux concentration of opposite polarity (not shown); this is analogous to the corresponding potential field.

The rise of the apex is initially exponential, reaching $0.3 V_{A 0}$ by $t \approx 12 \tau_{A}$, followed by an approximately linear rise (with slowly decreasing velocity), qualitatively similar to the two rise profiles shown below in Figure 6 (Section 2.2). This is the typical behavior of fast CMEs and consistent with the 18 April 2001 eruption, which showed a fast linear rise after the occulted short main acceleration phase. We note that the flux rope enters the height range of the torus instability in the course of its main acceleration. This instability, which is a form of the lateral kink (Kliem and Török, 2006), eventually drives the flux rope to a full eruption.

The isosurfaces of the current density plotted in Figure 4 show the development of current sheets, in addition to the kinking, interaction, and reformation of an approximately straight current channel in the core of the flux rope. The 


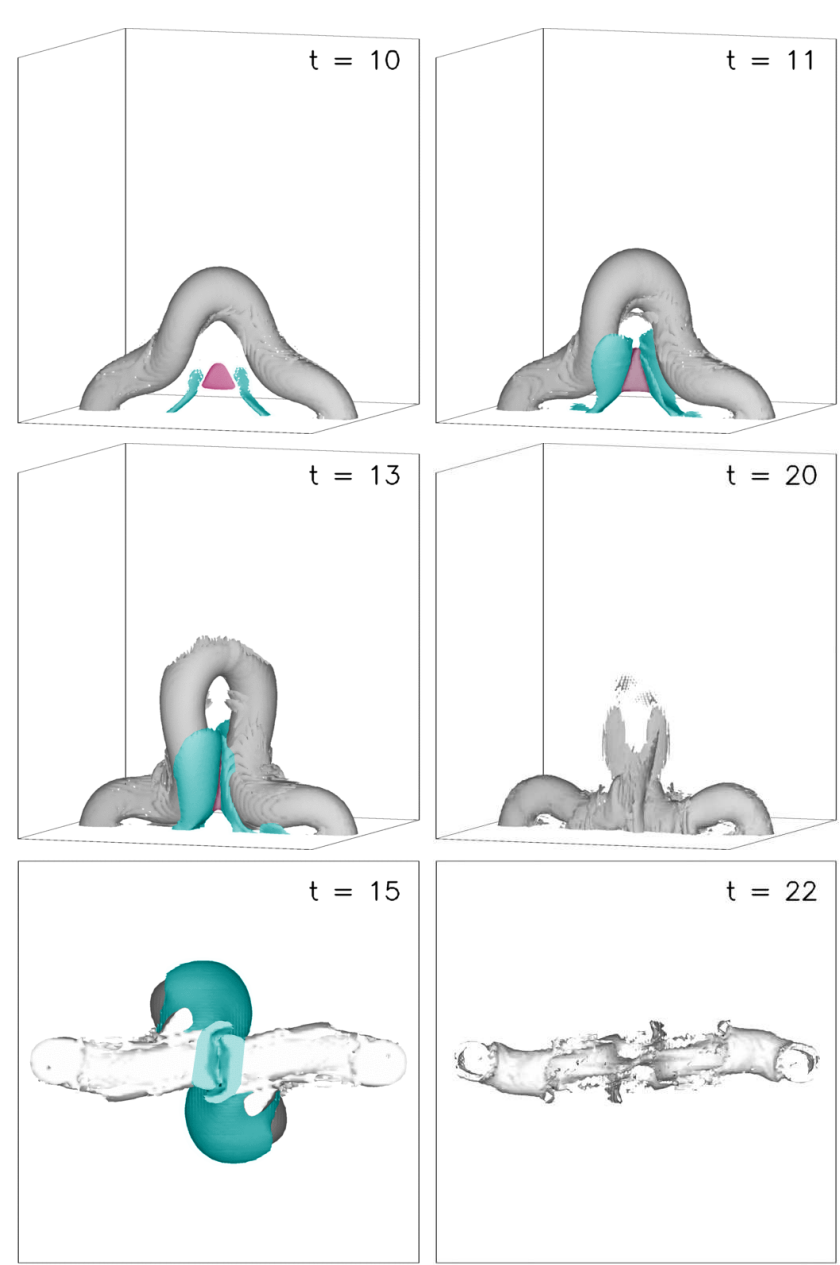

Figure 4. Isosurfaces of current density, at $10 \%$ of the peak current density in each snapshot, showing the kinking, reconnection, and reformation of the current channel (gray) in the core of the flux rope which is displayed in Figures 2 and 3 . In addition, the formation of the vertical (flare) current sheet (red) and of the lower parts of the helical current sheet (cyan) are apparent in panels 1 and 2. Panel 3 shows the temporary formation of a double current sheet from two sections of the helical current sheet between the approaching flux rope legs just before the legs reconnect. Upward views through the bottom of the box in panels 5 and 6 show the bottom edge of the squeezed current sheet (cyan) between the light-gray horizontal sections of the flux rope legs, the dark-cyan underside of the helical current sheet that wraps around the upper part of the flux rope legs $(t=15)$, and the current flow through the reconnected horizontal sections of the flux rope legs $(t=22)$. The inner subvolume $4^{3}$ is displayed.

first panel displays the onset of current density steepening in the vertical (flare) current sheet below the rising flux rope apex (red triangular structure) and in the helical current sheet (two oblique cyan layers near the knees of the bent channel). While the vertical current sheet exists only below the rising current channel, the helical current sheet actually wraps around the whole channel, passing over the apex. Only its lower sections are visible in these isosurface plots, since the current 
density in the helical sheet decreases rapidly with height as a consequence of the rapidly decreasing flux density of the ambient field. Therefore, the helical displacement of the upper parts of the flux rope cannot pile up much flux in front of the interface to the ambient field, where the helical current sheet forms. (See, e.g., Fig. 7 in Arber, Longbottom, and van der Linden, 1999, Fig. 4 in Gerrard et al., 2001, and Fig. 3 in Török, Kliem, and Titov, 2004 for isosurface plots that show the complete helical current sheet formed around kinking flux ropes which are embedded in less inhomogeneous field, and Fig. 10 in Paper I for field line plots of the helical current sheet. See also Lionello et al., 1998 for the formation of the helical current sheet in a representative range of kink-unstable flux rope equilibria.)

The second and third panels in Figure 4 show how the approaching legs of the kinking rope squeeze the two layers of the helical current sheet and the vertical current sheet between them into a vertical double current sheet. The fourth panel demonstrates that the double current sheet and the legs of the kinking current channel reconnect, reforming a low-lying current channel (flux rope).

Depending on the extent of the kink and on how much the rope rises during the kink, one can expect the two legs of the tube to hit each other at somewhere between right angles and an antiparallel orientation. Linton, Dahlburg, and Antiochos (2001) explored how reconnection would happen at high twist $(\Phi=20 \pi)$ for such collisions. Considering an idealized set-up with periodic boundary conditions and magnetically isolated, straight twisted flux tubes brought together by a prescribed slow stagnation-point flow, these authors found that antiparallel flux tubes of like helicity bounce off each other with hardly any reconnection occurring, hence they termed this case "bounce interaction." This is a natural consequence of the fact that the fields at the surface of the flux tubes, which are dominated by the azimuthal component for such strong twist, are approximately parallel where the tubes come into contact. They found a similar bounce interaction when the tubes collide at right angles with the same signs of twist helicity and crossing helicity (i.e., RR2: right handed tubes at a collision angle of $2 \pi / 8$ or LL6: left handed tubes at an angle of $6 \pi / 8$, in the nomenclature of Linton, Dahlburg, and Antiochos, 2001). On the other hand, they found that the tubes undergo significant reconnection at right angles, and in fact tunnel through each other, if the twist helicity and crossing helicity are of opposite signs (RR6 or LL2). Linton (2006) then showed that this tunnel interaction can occur for such collisions at twists as low as $\Phi=9 \pi$, which equals the twist of the kink simulation shown in Figures 2 团 here. This raises the interesting question of whether such a tunnel reconnection could occur in the kink-induced reconnection simulations we present here. Unfortunately, it turns out this can not happen, as the kink always gives a crossing number which is unfavorable for tunnel reconnection. The kink instability in a left hand twisted flux tube induces left hand writhing, which in turn gives a left handed flux tube crossing. Thus the collision of flux tube legs induced by the kink instability gives the same sign of crossing number and twist (LL6 or RR2), and can not induce tunneling reconnection.

In spite of this, we do find significant reconnection in this kink-induced collision. This, we interpret, is due to the fact that the twist here is initially less 
than half that of the tubes studied by Linton, Dahlburg, and Antiochos (2001), and the kink and eruption of the tubes subsequently lower it even further. This allows the toroidal field to dominate over the twist field at the point where the tubes collide. The toroidal field is antiparallel for an antiparallel tube collision, and is at right angles for a right angle collision. Both orientations are favorable for reconnection, and so significant flux tube reconnection is generated by these collisions. Furthermore, the reconnection is here driven and enforced by the overall helical deformation of the flux rope, which is a fast process, since the helical kink is an ideal MHD instability. The current channels of diameter $2 a \approx 0.4$ merge completely within $\approx 8 \tau_{A}$ (between $t \approx 12.5$ and $t \approx 20$ ), corresponding to a high reconnection inflow Alfvén Mach number of $\approx 0.05$.

A further difference to the simulations in Linton, Dahlburg, and Antiochos (2001) is given by the presence of the helical current sheet. This sheet and the current flowing in it wrap around the current channel in a helical manner, with the overall direction in the sheet being opposite to the direction of current flow in the channel. When the two layers of the helical current sheet are squeezed together between the approaching, nearly vertical legs of the channel, they are nearly antiparallel (see the third panel of Figure 4). Such a double current sheet is known to be susceptible to the double tearing mode. Previous two-dimensional simulations of this mode had sufficient spatial resolution to demonstrate that it creates a chain of magnetic islands in each layer, with the island growth in each layer facilitating the growth in the other layer. This leads to fast reconnection which depends only weakly on the resistivity (e.g., Pritchett, Lee, and Drake, 1980, Matthaeus and Lamkin, 1986, Wang et al., 2007). As in a single current sheet, the islands in each layer merge subsequently, driven by the coalescence instability. The merged islands - plasmoids - typically oscillate and are eventually ejected at high speed along the sheet if no symmetry is prescribed (Tajima et al., 1987 Schumacher and Kliem, 1996; Magara, Shibata, and Yokoyama, 1997;:Bárta, Vrsňak, and Karlický, 2008). Such acceleration of plasmoids along the sheet is amplified in the double tearing mode by the repelling force between neighboring plasmoids in different layers because the currents are oppositely directed (Matthaeus and Lamkin, 1986). Hence, the double tearing mode has the potential to support fast reconnection, involving plasmoid formation and ejection, when two layers of the helical current sheet are squeezed between the approaching legs of a kinking flux rope.

Far higher spatial resolution than is available in our simulation would be required to resolve the details of the reconnection between the two layers of the helical current sheet and between the flux rope legs in three dimensions. Therefore, we cannot here model the double tearing mode or other processes that may form plasmoids or other structures capable to confine the accelerated particles in compact radiation sources, as observed in the 18 April 2001 flare. Rather, we have to rely on the basic knowledge reviewed above. It must be kept in mind, however, that the rapid driving of the reconnection in the double current sheet by the approaching flux rope legs may modify the evolution of the double tearing mode with respect to the quoted numerical simulations, which considered a double current sheet without external driving.

The reconnection induces a peak parallel electric field drop equal to the rate of flux change (Hesse, Forbes, and Birn, 2005), which is directly available to 
particle acceleration, and a perpendicular electric field of order $E_{\perp}=0.05 V_{A} B$, part of which can be tapped by particles drifting in the inhomogeneous reconnection volume (e.g., Ambrosiano et al., 1988, Kliem, 1994, Drake et al., 2006. Karlický and Bárta, 2007). Assuming, for example, a field strength $B=$ 100 Gauss and a particle density $n=10^{10} \mathrm{~cm}^{-3}$, we have $V_{A 0} \approx 2000 \mathrm{~km} \mathrm{~s}^{-1}$ and $E_{\perp} \sim 1 \mathrm{kV} \mathrm{m}^{-1}$. Assuming in addition an initial flux rope height of $30 \mathrm{Mm}$, the simulation results scale to a flux in the current channel of $\sim 10^{19} \mathrm{Mx}$ (a factor $\lesssim 10$ lower than the flux in the whole flux rope), reconnected in $\sim 110 \mathrm{~s}$, which yields a parallel potential of $\sim 1 \mathrm{GV}$. Both are huge values, more than sufficient to enable the acceleration of particles to the observed energies.

The region of reconnection between the flux rope legs does not participate in the fast rise of the flux rope's upper part, rather it remains in the range of heights where it commences, $z \lesssim 1$, with a slight tendency to shift downwards from the legs' initial touching point (Figures 14 4). This is in qualitative agreement with the observations of the 18 April 2001 eruption described in Paper I, which indicate that leg-leg reconnection commenced at about the time the crossing point of the legs had appeared at the limb, i.e., at a height slightly above $\sim 0.1 R_{\odot}$, and that the region of interaction subsequently remained near the limb or even retracted slightly (see also Figure 7 below). However, the heights of the observed crossing point and of the commencing reconnection in the simulation differ by a factor $\sim 1.5$. This value can roughly be estimated from the length of the polarity inversion line between the main flux concentrations in AR 9415 before it rotated beyond the limb, about $10^{\circ}$ or $\sim 0.2 R_{\odot}$, which should be comparable to the distance, $D$, between the footpoints of the erupting flux loop. The observationally indicated height range of leg-leg reconnection thus is $h_{\mathrm{r}} \lesssim D / 2$, while the simulation above, with $D=3.3$, yields $h_{\mathrm{r}} \lesssim D / 3$. This moderate disagreement can be resolved by the inclusion of a second process that causes the flux to rise higher before it starts to reconnect. Candidate processes are an accelerated slow-rise phase, for example due to slow photospheric motions which lead to an amplifying inflation of the current-carrying coronal field (e.g., Török and Kliem, 2003, Aulanieret al., 2010), as well as any other CME driver, for example another instability. We consider the latter possibility in the following subsection.

High twist and leg-leg reconnection do not always lead to ejective behavior. When the simulation is repeated with a stronger line current (as in Török and Kliem, 2005) but otherwise identical parameters, a failed eruption is found. Reconnection commences between the approaching legs of the rope, as decribed above, but soon thereafter it also commences in the section of the helical current sheet above the flux rope apex, where the current density is now much higher due to stronger flux pileup. The latter reconnection, described in Török and Kliem (2005), cuts the rope at its top, thus preventing an ejective evolution.

\subsection{Helical Kink Supported by the Torus Instability}

Here we describe a flux rope that is susceptible to the helical kink mode and to the torus instability from the beginning. The torus instability (Kliem and Török, 2006) is the process behind the well-known catastrophe of a flux rope-arcade configuration (van Tend and Kuperus, 1978; Forbes and Isenberg, 1991; Lin et al., 1998). 


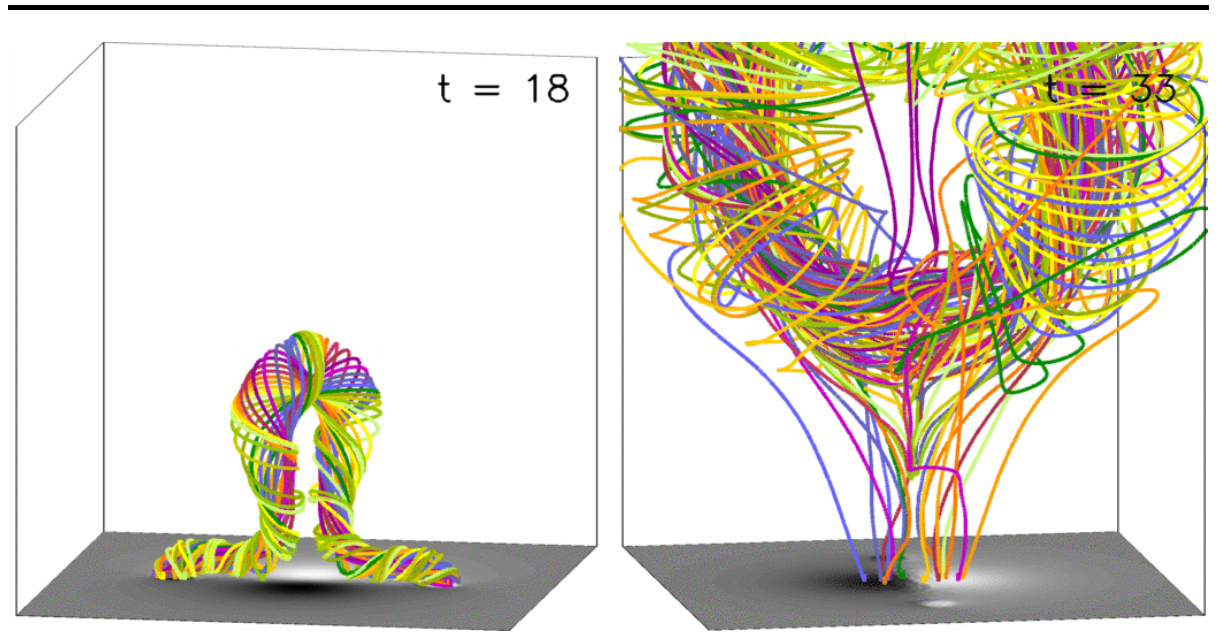

Figure 5. Left: Field lines of a kink and torus unstable flux rope of initial twist $\Phi=8 \pi$ just before leg-leg reconnection commences, showing that the height range of leg approach is somewhat enlarged in comparison with the case shown in Figures 24 due to the additional upward acceleration by the torus instability. Right: The same configuration at a later time showing that part of the flux in the escaping upper part of the original flux rope produces closed loops as a result of the reconnection between the legs, while the other part has reconnected with the ambient field. The field line start points lie in a circle of radius $\approx(2 / 3) a(t)$ centered at the flux rope's apex. The inner subvolume of the box of size $6^{3}$ is shown. The views differ by a rotation of $70^{\circ}$ about the $z$ axis.

It provides an additional upward acceleration of the flux rope, raising the height range of leg-leg reconnection due to the helical kink mode. In order to make the initial equilibrium considered in Section 2.1 also torus unstable, one has to modify the external poloidal field such that it falls off more rapidly with height, or to reduce the stabilizing influence of line-tying. Let us first describe a simulation that realizes the former option by reducing the distance of the external poloidal field sources to $L=0.25$. The twist is reduced slightly to bring the time scales of the two instabilities closer together (so that the torus instability can influence the flux rope sufficiently early in the process of helical deformation), but it is still kept at a rather high value of $\Phi=8 \pi$. The resulting evolution of the flux rope is very similar to the case described in Section 2.1. except that the upper part of the rising loop is now stretched upward somewhat stronger, as shown in the left panel of Figure 5. The reconnection commences at somewhat greater heights in this run, in the range $z \approx 1$, and the current sheets between the flux rope legs are squeezed in the height range up to $z \approx 1.5$. This remains the range of reconnection between the legs as the eruption evolves, as indicated by the shape of the field lines in the right panel of Figure 5. This figure also proves the leg-leg reconnection by showing that part of the flux in the upper part of the broken rope closes with itself. The other part reconnects with the ambient field, forming new, less sharply defined legs.

As in the run in Section 2.1, the rise profile of the flux rope apex shows the two phases typical of fast CMEs: a rapid exponential rise to the peak velocity of nearly $0.4 V_{A}$ is followed by a linear further rise with very slowly decreasing velocity (Figure 6). The main part of the original flux in the legs of the rope 


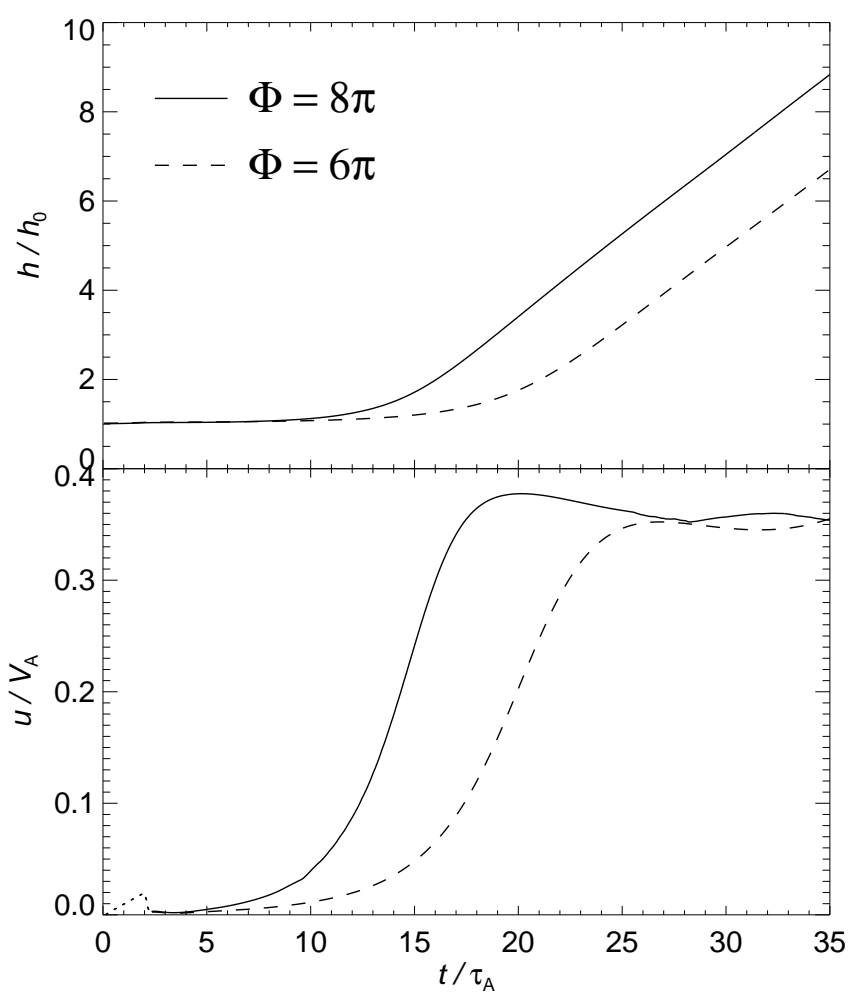

Figure 6. Rise profiles of the fluid element at the flux rope apex for the two simulations of Section 2.2 that are compared to the $17 \mathrm{GHz}$ images of the flare on 18 April 2001 in Figure 7 below (solid lines) and in Figure 9 of Paper I (dashed lines). The initial velocity perturbation applied at the apex is shown dotted.

reconnects in the interval $t \approx(18-25) \tau_{A}$, i.e., shortly after the peak acceleration of the ejection, similar to the run in Section 2.1 and exactly as inferred for the 18 April 2001 flare (see Paper I). Reconnection involving the newly formed legs and the ambient field continues subsequently at a lower rate until the run is terminated.

This run reproduces the key features of the evolving shape of the microwave source in the 18 April 2001 flare. Figure 7 displays, from top to bottom, the top section of a flux loop, the transition to concave-upward shape, the initially slim ellipse, and finally the considerably expanded and presumably further rotated ellipse, whose bottom stays close to the limb.

An even better agreement with the observed shape was obtained in another run which started from a less twisted rope $(\Phi=6 \pi)$, thus reducing the growth rate of the helical kink further and permitting a stronger influence of the torus instability. The external poloidal field in this run was chosen as in Section 2.1 and the torus instability can grow because the line-tying is reduced by placing the rope at a higher position $(R=1.1, d=0.1)$, giving it a nearly semicircular initial shape. The comparison with the microwave images is shown in Figure 9 of Paper I in the same format as in Figure 7 here. The rise profile is included in Figure 6 It 


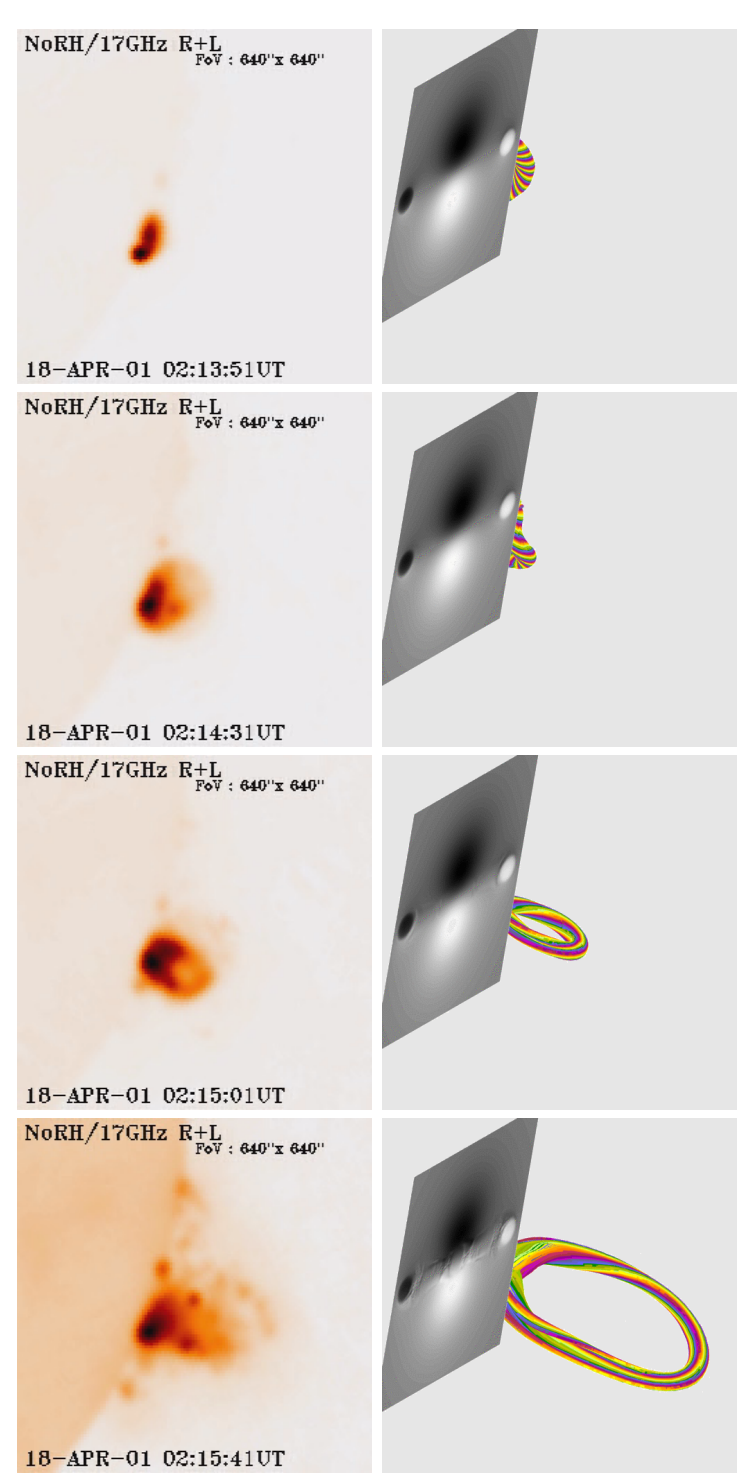

Figure 7. Comparison of the evolving $17 \mathrm{GHz}$ source in the 18 April 2001 eruption with the shape of the kink-unstable flux rope of initial twist $\Phi=8 \pi$ shown also in Figure 5 The simulated flux rope is displayed at the same viewing angle as the partly occulted microwave source (tilted away from the observer by $26^{\circ}$, so that the magnetogram, $B_{z}(x, y, 0, t)$, is seen from below the simulation box). The field lines outline the core of the flux rope at $t=0,12$, 17 , and $24 \tau_{A}$.

shows the same two-phase velocity profile and the same timing of reconnection between the flux rope legs $\left(t \gtrsim 23 \tau_{A}\right)$ relative to the main acceleration phase as the previous two runs.

This third run in particular brings observation and simulation in reasonable quantitative agreement, resolving the moderate discrepancy in the height of the 
crossing/reconnection-onset point discussed in Section 2.1. As mentioned there, further possibilities exist to raise the height of leg-leg reconnection onset in the numerical modeling; however, we refrain from further pursuing the parametric study of this minor aspect.

\section{Discussion}

The analysis of the microwave data of the eruptive flare on 18 April 2001 and the comparison with the above MHD simulations suggest that the eruption involved a kink-unstable flux rope that had a high twist of $\Phi \gtrsim 6 \pi$. This yields a coherent framework to understand the inverse gamma shape of the rising main microwave source, as well as the triggering and ejection of superimposed compact sources that likely emerged from the crossing point and moved upward to the top of the main source.

The inferred twist lies several times above the often quoted threshold of $2.49 \pi$ for the helical kink instability of a line-tied flux rope (Hood and Priest, 1981; Einaudi and Van Hoven, 1983), so that the question arises how such a high value can occur. It can likely be acquired by the flux rope in the eruption process itself, by adding current-carrying flux to the rising and growing rope through reconnection under it. This addition of flux is well established theoretically (e.g., Lin and Forbes, 2000; Lin, Raymond, and van Ballegooijen, 2004) and observationally (e.g., Qiu et al., 2004). Resulting twist values far exceeding the above threshold have been inferred from some interplanetary CMEs (Gulisano et al., 2005, Qiu et al., 2007), but the corresponding coronal values remain elusive.

It is also possible that the initial equilibrium had acquired the required twist. The threshold found by Hood and Priest (1981) and Einaudi and Van Hoven (1983) is the lowest possible threshold, valid only for a uniformly twisted (GoldHoyle) flux rope of infinite radial extent. Several factors influence the threshold of the instability, among them the embedding of the flux rope in strong external current-free field, the inhomogeneity of the radial twist profile and the radius of the rope. In particular, it has been shown by Baty (2001) that the threshold can lie at twists of $10 \pi$ and higher if the radius of the rope becomes smaller than the pitch length of the twisting field lines. With regard to the acquired twist, the event on 18 April 2001 appears to be atypical, belonging to a minority of cases with very high twist. Other candidate cases for such and even somewhat higher twist are described in Vršnak, Ruždjak, and Rompolt (1991), Romano, Contarino, and Zuccarello (2003), and in Cho et al. (2009).

Cho et al. (2009) suggested that a kinking flux rope with reconnecting legs caused an eruptive X-class limb flare on 18 August 2004. They found evidence for the break-up of a kinked loop at the projected crossing point of the legs. Hot EUV-emitting plasma was produced at low heights up to the breaking point, presumably by reconnection, while the top part of the loop was further accelerated and evolved into a CME. An outward moving compact coronal hard $\mathrm{X}$-ray source appeared at the position and time of the break-up inferred from EUV and $\mathrm{H} \alpha$ images; this source was seen at higher energies than the well known 
coronal sources formed at or above the top of soft X-ray loops in some events (e.g., Sui and Holman, 2003). These findings are analogous to the dynamics of the microwave and hard X-ray sources and to the spectral extent of the hard X-ray source in the 18 April 2001 flare discussed in Paper I, and they are in line with the simulations presented above.

The quick reformation of a flux rope below the reconnecting legs of the original rope can explain the quick reformation of filaments after some eruptions, for example, in the 19 July 2000 event investigated by Romano, Contarino, and Zuccarello (2003).

Further possible cases of reconnection at or near the crossing point of rising kinked filaments were presented in Ji et al. (2003) and Alexander, Liu, and Gilbertl (2006) for an event on 27 May 2002 and in Liu and Alexander (2009) for events on 12 June 2003 and 10 November 2004. These authors describe hard X-ray sources near the projected crossing point of the legs in addition to footpoint sources, and the latter two papers suggest leg-leg reconnection as one possible mechanism of the underlying particle acceleration. No break-up of the kinked filaments at the crossing point occurred, however, and the shapes are suggestive of a dominantly single helical $\left(k^{\prime} \sim 1\right)$ kink. The kinking of twisted flux was modeled for the first and third of these events in Török and Kliem (2005) and Williams et al. (2005), respectively, assuming a twist of $5 \pi$ in both cases, which led to a $k^{\prime} \sim 1$ kink. It is likely that leg-leg interaction occurred in the event on 27 May 2002, which showed the clearest association of the X-ray source with the crossing point, as a consequence of the failed nature of the eruption and the cut up of the filament at its top. We are preparing a corresponding simulation study to detail this suggestion. For the other two events, which were ejective, we believe that reconnection in the helical current sheet has likely played a role (partly similar to our suggestion here), but that the conjecture of leg-leg reconnection requires further study and justification.

\section{Summary and Conclusion}

MHD simulations of the helical kink instability of arched, line-tied flux ropes demonstrate that the legs of the flux rope reconnect with each other if perturbations with two helical turns, i.e., normalized axial wavenumbers $k^{\prime} \sim 2$, are dominant. This requires twists of $\approx 6 \pi$ or higher.

Leg-leg reconnection can cause a complete break-up of the original rope. The upper part of the original rope can evolve into a CME or stay confined in the corona. Its fate depends on the overlying field, as for less twisted erupting flux ropes (see Roussev et al., 2003; Török and Kliem, 2005, Kliem and Török, 2006). The lower parts can form a new flux rope connecting the footpoints of the original rope. The new rope is still considerably twisted. The break-up reconnection is found to involve reconnection with the ambient flux in all simulations, resulting in new connections of the rising upper part of the kinked rope to the footpoints of originally ambient flux. Furthermore, the downward reconnection outflow pushes the reformed flux rope towards the bottom plane, where it reconnects and breaks up a second time. The resulting two major flux bundles, 
which connect the footpoints of the original flux rope with the corresponding major ambient flux concentration (sunspot) of opposite polarity, represent a state close to the potential field of the given flux distribution in the bottom plane (illustrated, e.g., in Figs. 3 and 8 of Valori et al., 2010.) The simulations also show that the region of reconnection between the legs of the highly kinked flux rope stays low in the coronal volume and that this reconnection commences just after the main acceleration of the flux rope's upper part.

Such dynamics are in overall agreement with the flare on 18 April 2001 studied in Paper I, supporting the interpretation that the microwave source was formed by a flux rope which was destabilized by the helical kink mode at the onset of the eruption's main acceleration phase and experienced leg-leg reconnection in the nonlinear development of the instability. The occurrence of leg-leg reconnection argues against a significant role for the shear field component in the writhing of the erupting flux because this effect pushes the legs of the rope apart.

A quantitative difference relative to the observations is given by the fact that the crossing point of the inverse gamma shaped microwave source rose to a height of about half the footpoint distance before the reconnection commenced, while the simulations yield a height of about one third the footpoint distance if the eruption is solely driven by the helical kink mode. This indicates that the initial rise, at least up to the height where the reconnection commenced, involved a second process in addition to the helical kink instability. The data do not provide clues to the nature of that process, which could have been an accelerated slow-rise phase or any of the triggers of fast rise discussed in current CME models. Simulations that include the torus instability in addition to the double helical kink mode, as a representative model, resolve the discrepancy.

The approach of the flux rope legs squeezes sections of the helical current sheet associated with the kink mode into a temporary double current sheet between them. This configuration is susceptible to the double tearing mode, which is known to facilitate fast reconnection and the formation of moving plasmoids through subsequent island coalescence. However, the limited resolution of our simulations does not permit us to determine whether this mode did actually occur. Driven by the kink instability, the double current sheet and the flux rope legs reconnect essentially completely and at a high rate, with the Alfvén Mach number of the reconnection inflow reaching $\approx 0.05$.

The path of the moving compact microwave and hard X-ray sources along the upper part of the inverse gamma source and the coalescence of multiple blobs into a final compact microwave source at the top of the inverse gamma source in the 18 April 2001 flare are consistent with plasmoid formation and propagation in the helical current sheet formed by the $m=1$ kink instability. The alternative assumption of formation by reconnection in the vertical current sheet of the standard flare model appears to be incompatible with these observations (see Paper I).

These findings lead us to conclude that the eruptive flare on 18 April 2001 was likely caused — not exclusively but to a large part — by the helical kink instability of a highly twisted flux rope.

Acknowledgements We thank M. Shimojo for the Nobeyama Radioheliograph maps included in Figure 7 and T. G. Forbes for discussions of the 
instabilities considered here. This study was supported by the DFG, by an STFC Rolling Grant, by NASA Grants NNH06AD58I and NNX08AG44G, and by Grant 300030701 of the Grant Agency of the Czech Academy of Sciences. Financial support by the European Comission through the SOLAIRE network (MTRM-CT-2006-035484) is gratefully acknowledged. The research leading to these results has received funding from the European Commission's Seventh Framework Programme (FP7/2007-2013) under the grant agreement n 218816 (SOTERIA project, www.soteria-space.eu).

\section{References}

Alexander, D., Liu, R., Gilbert, H.R.: 2006, Astrophys. J. 653, 719.

Ambrosiano, J., Matthaeus, W.H., Goldstein, M.L., Plante, D.: 1988, J. Geophys. Res. 93, 14383.

Arber, T.D., Longbottom, A.W., van der Linden, R.A.M.: 1999, Astrophys. J. 517, 990.

Aulanier, G., Török, T., Démoulin, P., DeLuca, E.E.: 2010, Astrophys. J. 708, 314.

Bárta, M., Büchner, J., Karlický M.: 2010, Adv. Space Res. 45, 10.

Bárta, M., Vrsňak, B., Karlický, M.: 2008, Astron. Astrophys. 477, 649.

Baty, H.: 2001, Astron. Astrophys. 367, 321.

Birn, J., Forbes, T.G., Hesse, M.: 2006, Astrophys. J. 645, 732.

Cho, K.-S., Lee, J., Bong, S.-C., Kim, Y.-H., Joshi, B., and Park, Y.-D.: 2009, Astrophys. J. 703, 1.

Drake, J.F., Swisdak, M., Che, H., Shay, M.A.: 2006, Nature 443, 553.

Einaudi, G., Van Hoven, G.: 1983, Solar Phys. 88, 163.

Fan, Y.: 2005, Astrophys. J. 630, 543.

Fan, Y., Gibson, S.E.: 2003, Astrophys. J. 589, L105.

Forbes, T.G., Isenberg, P.A.: 1991, Astrophys. J. 373, 294.

Gerrard, C.L., Arber, T.D., Hood, A.W., Van der Linden, R.A.M.: 2001, Astron. Astrophys. 373, 1089

Gibson, S.E., Fan, Y., Mandrini, C., Fisher, G., Démoulin, P.: 2004, Astrophys. J. 617, 600. Gilbert, H.R., Alexander, D., Liu, R.: 2007, Solar Phys. 245, 287.

Green, L.M., Kliem, B., Török, T., van Driel-Gesztelyi, L., Attrill, G.D.R.: 2007, Solar Phys. 246, 365.

Gulisano, A.M., Dasso, S., Mandrini, C.H., Démoulin, P.: 2005, J. Atmos. Sol. Terr. Phys. 67, 1761.

Hesse, M., Forbes, T.G., Birn, J.: 2005, Astrophys. J. 631, 1227.

Hood, A.W., Priest, E.R.: 1981 Geophys. Astrophys. Fluid Dynamics 17, 297.

Isenberg, P.A., Forbes, T.G.: 2007, Astrophys. J. 670, 1453.

Ji, H., Wang, H., Schmahl, E.J., Moon, Y.-J., Jiang, Y.: 2003, Astrophys. J. 595, L135.

Karlický, M., Bárta, M.: 2007, Astron. Astrophys. 464, 735.

Karlický, M., Kliem, B.: 2010, Solar Phys., in press (Paper I).

Kliem, B.: 1994, Astrophys. J. Suppl. Ser., 90, 719.

Kliem, B., Török, T.: 2006, Phys. Rev. Lett. 96, 255002.

Kliem, B., Titov, V.S., Török, T.: 2004, Astron. Astrophys. 413, L23.

Lin, J., Forbes, T.G.: 2000, J. Geophys. Res. 105, 2375.

Lin, J., Forbes, T.G., Isenberg, P.A., Démoulin, P.: 1998, Astrophys. J. 504, 1006.

Lin, J., Raymond, J.C., van Ballegooijen, A.A.: 2004, Astrophys. J. 602, 422.

Linton, M.G.: 2006, J. Geophys. Res. 111, A12S09, doi:10.1029/2006JA011891.

Linton, M.G., Dahlburg, R.B., Fisher, G.H., Longcope, D.W.: 1998, Astrophys. J. 507, 404.

Linton, M.G., Dahlburg, R.B., Antiochos, S.K.: 2001, Astrophys. J. 553, 905.

Linton, M.G., Longcope, D.W., Fisher, G.H.: 1996, Astrophys. J. 469, 954.

Lionello, R., Velli, M., Einaudi, G., Mikić, Z.: 1998, Astrophys. J. 494, 840.

Liu, R., Alexander, D.: 2009, Astrophys. J. 697, 999.

Magara, T., Shibata, K., Yokoyama, T.: 1997, Astrophys. J. 487, 437.

Matthaeus, W.H., Lamkin, S.L.: 1986, Phys. Fluids 29, 2513.

Metcalf, T.R., De Rosa, M.L., Schrijver, C.J., et al.: 2008, Solar Phys. 247, 269.

Min, S., Chae, J.: 2009, Solar Phys. 258, 203. 
Pevtsov, A.A., Canfield, R.C., McClymont, A.M: 1997, Astrophys. J. 481, 973.

Pritchett, P.L., Lee, Y.C., Drake, J.F.: 1980, Phys. Fluids 23, 1368.

Qiu, J., Wang, H., Cheng, C.Z., Gary, D.E.: 2004, Astrophys. J. 604, 900.

Qiu, J., Hu, Q., Howard, T.A., Yurchyshyn, V.B.: 2007, Astrophys. J. 659, 758.

Romano, P., Contarino, L., Zuccarello, F.: 2003, Solar Phys. 214, 313.

Roussev, I.I., Forbes, T.G., Gombosi, T.I., Sokolov, I.V., DeZeeuw, D.L., Birn, J.: 2003, Astrophys. J. 588, L45.

Rust, D.M.: 2003, Adv. Space Res. 32, 1895.

Rust, D.M., Kumar, A.: 1996, Astrophys. J. 464, L199.

Rust, D.M., LaBonte, B.J.: 2005, Astrophys. J. 622, L69.

Sakurai, T.: 1976, Pub. Astron. Soc. Japan 28, 177.

Schrijver, C.J., Elmore, C., Kliem, B., Török, T., Title, A.M.: 2008a, Astrophys. J. 674, 586.

Schrijver, C.J., De Rosa, M.L., Metcalf, T., et al.: 2008b, Astrophys. J. 675, 1637.

Schumacher, J., Kliem, B.: 1996, Phys. Plasmas 3, 4703.

Sui, L., Holman, G.D.: 2003, Astrophys. J. 596, L251.

Tajima, T., Sakai, J., Nakajima, H., Kosugi, T., Brunel, F., Kundu, M.R.: 1987, Astrophys. J. 321, 1031.

Titov, V.S., Démoulin, P.: 1999, Astron. Astrophys. 351, 707.

Török, T., Kliem, B.: 2003, Astron. Astrophys. 406, 1043.

Török, T., Kliem, B.: 2005, Astrophys. J. 630, L97.

Török, T., Kliem, B., Titov, V.S.: 2004, Astron. Astrophys. 413, L27.

Valori, G., Kliem, B., Török, T., Titov, V.S.: 2010, Astron. Astrophys., in press, e-print arXiv:1005.0254

van Tend, W., Kuperus, M.: 1978, Solar Phys. 59, 115.

Vršnak, B.: 2001, J. Geophys. Res. 106, 25249.

Vršnak, B., Ruždjak, V., Rompolt, B.: 1991, Solar Phys. 136, 151.

Vršnak, B., Ruždjak, V., Rompolt, B., Roša, D., Zlobec, P.: 1993, Solar Phys. 146, 147.

Wang, Z.X., Wang, X.G., Dong, J.Q., Lei, Y.A., Long, Y.X., Mou, Z.Z., Qu, W.X.: 2007, Phys. Rev. Lett. 99, 185004.

Williams, D.R., Harra, L.K., Brooks, D.H., Imada, S., Hansteen, V.H.: 2009, Pub. Astron. Soc. Japan, 61, 493.

Williams, D.R., Török, T., Démoulin, P., van Driel-Gesztelyi, L., Kliem, B.: 2005, Astrophys. J. 628, L163.

Zhou, G. P., Wang, J. X., Zhang, J., Chen, P. F., Ji, H. S., Dere, K.: 2006, Astrophys. J. 651, 1238. 